\title{
Trend and prevalence of transfusion transmitted infections among blood donors in rural teaching institute, south India
}

\author{
Leena MS ${ }^{1}$, Mohd. Shafee ${ }^{2}$ \\ ${ }^{1}$ Department of Pathology, CAIMS, Bommakal, Karimnagar \\ ${ }^{2}$ Department of Community Medicine, CAIMS, Bommakal, Karimnagar
}

\section{Keywords: \\ Blood donor; \\ Seroprevalence; Transfusion; Infection}

\begin{abstract}
Background: The magnitude of transfusion transmitted infections (TTI) varies from country to country depending on TTI's load in that particular population. The aim of the study was to study the trend and prevalence of sero-markers among blood donors in one of the tertiary health center in south India.
\end{abstract}

Materials and Methods: A retrospective cross-sectional study was performed from 2004 to 2010 in a blood bank of teaching hospital in South India. All blood donors who presented to blood bank during the study period were included. Data regarding demography and serological tests were collected on predesigned proforma.

Results: There were a total of 6939 blood donors during study period. Out of these, 94(1.35\%) were positive for sero-markers for TTIs. The number of blood donors as well as sero-positivity increased from year 2004 to 2010 .

Conclusions: Trend and sero-prevalence of TTIs increased over period of time. Sero-positivity for TTIs decreased from year 2004 to 2010.

\section{INTRODUCTION}

Transfusion transmitted infections (TTIs) are a great concern of safety for patients. Since the starting of blood transfusion scientifically in early 1940s, various transfusion associated problems have come to the forefront for the scientific community. These include TTI, alloimmunization to various blood components and issues related to cold chain maintenance. TTI was first observed in the process of blood transfusion in late 1940 . Till early 1970, blood

\section{Correspondence:}

Dr. Leena $M S, M D$

Department of Pathology, CAIMS Campus, Bommakal, Karimnagar, India Tel: +919440929199

E-mail:mohdshafee2008@gmail.com bank personnel were only concentrating on a few blood borne infections like syphilis and viral hepatitis despite the constant awareness regarding the presence of multiple agents. ${ }^{1}$

A well organized Blood Transfusion Service (BTS) is a vital component of any health care delivery system. An integrated strategy for Blood Safety is required for elimination of TTIs and for provision of safe and adequate blood transfusion services to the people. The main component of an integrated strategy include collection of blood only from voluntary, non-renumerated blood donors, screening for all TTIs and reduction of unnecessary transfusion. ${ }^{2}$ 
The BTS in the country is highly decentralized and lacks many vital resources like manpower, adequate infrastructure and financial aspect. The standards of BTS vary from state to state, cities to cities and centre to centre. Many large hospitals and nursing homes do not have their own blood banks and this has led to proliferation of stand-alone private blood banks. ${ }^{2}$

The appropriate use of blood and blood products means the transfusion of safe blood products to treat a condition leading to significant morbidity or mortality that cannot be prevented or managed effectively by other means. Transfusion carries the risk of adverse reactions and transfusion transmissible infections. Plasma can transmit most of the infections present in whole blood and there are very few indications for its transfusion. Blood donated by family/replacement donors carries a higher risk of TTIs than blood donated by voluntary non-renumerated donors. Paid blood donors have the highest incidence and prevalence of TTIs. $^{3}$

Blood transfusion involves transfer of biological material from man to man. It is therefore unlikely to be totally free of the risk of the infections. The diseases transmitted by blood are HIV, hepatitis B and C, syphilis, malaria and infrequently cytomegalovirus, Epstein Barr virus, brucellosis etc. Preventing transmission of these infectious diseases through blood transfusion presents one of the greatest challenges of transfusion medicine. ${ }^{4}$ This study was aimed at identifying the status of transfusion transmitted diseases among healthy non-renumerated donors over a period of time so as to heighten the awareness of the infectious complications of blood transfusion.

\section{MATERIALS AND METHODS}

Study design and Study Period: This was a retrospective cross-sectional study carried out in one of the medical institute in south India. The records of blood donors who reported to blood bank from January 2004 to December 2010; were analyzed. The permission from head of the institution and clearance from Institutional Ethics Committee was obtained.

Enrolment and eligibility: All blood donors who reported to blood bank during study period and who satisfied the criteria for blood donation were included in this study.

Diagnostic setting: The blood bank staff keeps the record of demography, weight, haemoglobin status and results of serological test in a register. The testing protocol followed in the blood bank is after complete physical examination by blood bank medical officer; venous blood sample from all donors for testing HBsAg, HIV, HCV, VDRL and malaria. The slide agglutination test was used for VDRL screening. The positive VDRL was confirmed by Treponema Pallidum Heamagglutination (TPHA) test. The malarial parasite was examined on peripheral blood smear and confirmed by serology. The blood was allowed to clot for $50 \mathrm{~min}$ at room temperature and serum was separated after centrifugation. The serum sample was then subjected to serological test.

The kits applied for various test were manufactured by J. Mitra, New Dehi, BioStandard Diagnostics, Gurgaon, Haryana and Ensure Biotech, Hyderabad for HIV, HBV, $\mathrm{HCV}$, syphilis and malaria.

Statistical analysis: The statistical analysis was done by statistical software form www.OpenEpi.com by applying various statistical tests.

\section{RESULTS}

There were total 6939 blood donors during the study period. The donor group comprised predominantly of males with females accounting for $<4 \%$. Out of these, 94(1.35\%) were positive for sero-markers for TTIs. The numbers of blood donors have increased and sero-positivity for TTIs decreased from 2004 to 2010. This decrease in sero-positivity was found to be statistically significant $(\mathrm{P}=0.01293)$. Trend of TTIs among blood donors are shown in Table 1.

Regarding different TTIs, highest numbers of blood donors $(0.71 \%)$ were having Hepatitis B followed by HIV $(0.27 \%)$, Hepatitis C (0.14\%), Malaria (0.129\%) and Syphilis $(0.10 \%)$. The Hepatitis B had shown a linear increasing trend over a period of time whereas HIV was more in 2007 and 2010. Majority of Malaria cases were in the year 2010 (Table 2).

The majority of TTIs were found among blood donors (61.7\%) belonging to age group of 21-30 years followed by $31-40$ years of age group (28.72\%). Least number of TTIs were found among blood donors either aged below 21 years or more than 40 years $(\mathrm{X} 2=20.45, \mathrm{df}=1, \mathrm{P}<0.0001)$. Malaria cases were in the age group of $41-50$ years of age group. The blood donors belonging to age group 51-60 years were not having any TTIs (Table 3 ).

\section{DISCUSSION}

TTIs continue to be a big threat to the safety of blood supply more so in the developing and under developed countries. Viral infections are the major cause of morbidity and mortality in blood recipients. ${ }^{5}$ In this study in South India we found overall sero-prevalence of $1.35 \%$ of TTIs among healthy blood donors. The trend of sero-positivity increased from $0.043(3 / 6939)$ to $0.489(34 / 6939)$ over period of time from 2004 to 2010. When Extended Mantel-Haenszel ChiSquare for linear trend was applied, it was found that the sero-positivity increased significantly from 2004 to 2010 $(\mathrm{X} 2=6.18, \mathrm{df}=1, \mathrm{P}=0.01293)$. In Delhi, Makroo RN et al reported similar trend of increase in TTIs from 5.24/1000 in 1992 to $7.48 / 1000$ in $1993 .^{6}$ In contrast to the finding of 
Table 1: Trend of TTIs Among Blood Donors

\begin{tabular}{cccc}
\hline Year & Sero-positive* & Sero-negative & Total \\
\hline 2004 & $03(3.85)$ & $75(96.15)$ & $78(1.12)$ \\
2005 & $03(2.22)$ & $132(97.78)$ & $135(1.95)$ \\
2006 & $08(2.08)$ & $377(97.92)$ & $385(5.55)$ \\
2007 & $12(1.56)$ & $758(98.44)$ & $770(11.09)$ \\
2008 & $14(1.52)$ & $909(98.48)$ & $923(13.31)$ \\
2009 & $20(1.20)$ & $1649(98.80)$ & $1669(24.05)$ \\
2010 & $34(1.14)$ & $2945(98.86)$ & $2979(42.93)$ \\
\hline Total & $\mathbf{9 4}(\mathbf{1 . 3 5})$ & $\mathbf{6 8 4 5 ( 9 8 . 6 5 )}$ & $\mathbf{6 9 3 9 ( 1 0 0 )}$ \\
\hline
\end{tabular}

*sero-markers positive for HIV, HBV, HCV, Syphilis and Malaria.

Extended Mantel-Haenszel chi square for linear trend $=6.18, d f=1, P=0.01293$

Table 2: Trend of Different TTIs Among Blood Donors

\begin{tabular}{|c|c|c|c|c|c|c|}
\hline Year & No. of Donors & HBV & HIV & $\mathrm{HCV}$ & Malaria & Syphilis \\
\hline 2004 & $78(1.12)$ & $01(0.014)$ & - & - & $01(0.014)$ & $01(0.014)$ \\
\hline 2005 & $135(1.95)$ & $01(0.014)$ & $02(0.028)$ & - & - & - \\
\hline 2006 & $385(5.55)$ & $02(0.028)$ & $02(0.028)$ & $03(0.043)$ & - & $01(0.014)$ \\
\hline 2007 & 770 (11.09) & $08(0.115)$ & $04(0.058)$ & - & - & - \\
\hline 2008 & $923(13.31)$ & $06(0.086)$ & $03(0.043)$ & $03(0.043)$ & - & $02(0.028)$ \\
\hline 2009 & $1669(24.05)$ & $15(0.216)$ & $02(0.028)$ & $01(0.014)$ & - & $02(0.028)$ \\
\hline 2010 & $2979(42.93)$ & $16(0.230)$ & $06(0.086)$ & $03(0.043)$ & $08(0.115)$ & $01(0.014)$ \\
\hline Total & $6939(100)$ & $49(0.71)$ & $19(0.27)$ & $10(0.14)$ & $09(0.129)$ & $07(0.10)$ \\
\hline
\end{tabular}

Note: Numbers in parenthesis indicates percentage

Table 3: Age distribution of blood donors according to sero-markers

\begin{tabular}{|c|c|c|c|c|c|c|c|}
\hline \multirow{2}{*}{ Year } & \multirow{2}{*}{ No. of Donors } & \multicolumn{6}{|c|}{ Infective status of donors } \\
\hline & & HBV & HIV & HCV & Syphilis & Malaria & Total \\
\hline $18-20$ & 105 & - & - & 01 & - & - & 01 \\
\hline $21-30$ & 4589 & 34 & 13 & 03 & 05 & 03 & 58 \\
\hline $31-40$ & 2128 & 10 & 6 & 06 & 01 & 04 & 27 \\
\hline $41-50$ & 92 & 5 & - & - & 01 & 02 & 08 \\
\hline $51-60$ & 25 & - & - & - & - & - & - \\
\hline Total & 6939 & 49 & 19 & 10 & 07 & 09 & 94 \\
\hline
\end{tabular}

our study Kurl A et al in Ludhiana, reported declining trend of sero-markers from 1993 to $2003 .^{7}$ The sero-prevalence of TTIs found in this study was $1.35 \%$ which is less as compared to sero-prevalence of $1.63 \%$ found in Gangtok. ${ }^{8}$

In this study we found that highest numbers of blood donors were having Hepatitis B $(0.71 \%)$ followed by HIV $(0.27 \%)$, hepatitis C (0.14\%), malaria (0.129\%) and syphilis $(0.10 \%)$. The sero-prevalence was $<1 \%$ for all TTIs. Among these, highest prevalence was for hepatitis B but its prevalence was less as compared to $0.87 \%$ which was found in Jaipur, Rajasthan. ${ }^{9}$ Various studies reported that sero-prevalence of hepatitis B among blood donors was higher than HIV, HCV and syphilis. $4,6,7,9,10$

In India blood donors constitute the third main source of
HIV. ${ }^{4}$ The seroprevalence of HIV infection in the general population is $0.3 \%$. In our study the HIV seroprevalence was $0.27 \%$. In the year 2007 and 2010 majority of blood donors were seropositive for HIV. The reported seroprevalence is far less than other studies from various parts of India and Nepal. ${ }^{4,7-9,11,12}$

Most common viral hepatitis next to Hepatitis B is Hepatitis C. In this study the seroprevalence for Hepatitis $\mathrm{C}$ was $0.14 \%$ which is less as compared to the study done by Bagga PK et al. ${ }^{12}$ Other studies also reported higher seroprevalence of HCV than our study. ${ }^{4,6,7,9,10,12}$ In a study done in Aligarh seroprevalence of $\mathrm{HCV}$ was less than the present study. ${ }^{5}$

The seroprevalence of Syphilis was $0.10 \%$ in this study, which is less than the study of Srikirshna $(1.6 \%) .{ }^{4}$ The 
prevalence of Malaria was $0.129 \%$. Malaria positive cases in 2004 and 2010 were one and eight cases respectively (Table 2). During this period there was outburst of mosquito breeding places and simultaneously epidemic of Dengue fever was also going on in the region. There are very few studies available on prevalence of Malaria among blood donors. In Banglore, no donor from 8617 was found to be positive for Malaria. ${ }^{4}$

The seroprevalence of TTIs was significantly higher among blood donors in age group 21-40 years as compared to donors above 40 years $(\mathrm{X} 2=20.45, \mathrm{df}=1, \mathrm{P}<0.0001)$. Total 84 from 94 seropositive blood donors belonged to age group 21-40 years. This is an important finding that sexually active age group are having higher seroprevalence. In Jaipur, Sood $\mathrm{S}$ et $\mathrm{al}^{9}$ found that HIV was higher among age group from 31-40 years and Panda S in Orissa found that HIV and HCV was higher in age group of $21-30$ years. ${ }^{13}$

TTIs contribute a definite and serious complication of blood transfusion even with non-renumerated so called 'safe blood donors'. Taking into account the higher seropositivity among replacement donors, there should be motivation of public for voluntary donation and directed donation should be discouraged. As the magnitude of Hepatitis B is more than the HIV, HCV testing should also be routinely done. Inspite of all testing the risk of disease transmission through blood cannot be reduced to zero due to lack of tests which are $100 \%$ sensitive, difficulty in adopting the high sensitive test like DNA hybridization and PCR for bulk use, failure to detect those donors who are in the window period and also the potential for novel infectious agents. The increased testing will also result in loss of some safe donors due to false positivity and additional expense of testing to the patient. ${ }^{4}$

\section{CONCLUSION}

Seroprevalence of TTIs was found to be low which reflects the level of TTIs in the general population. The seroprevalence of TTIs was higher in sexually active blood donors. Hence, this study recommends that a three step approach towards safety of blood transfusion should include stringent donor screening, reliable screening tests and judicious use of blood and blood products.

\section{ACKNOWLEDGEMENTS}

The author acknowledges to all blood donors who donated blood for saving the lives of patients.

\section{REFERENCES}

1. Choudhary N. Transfusion transmitted infections: How many more? Asian J Trans Sci 2010;4:71-2.

2. National Blood Policy 2003, National AIDS Control Organisation, Ministry of Health \& Family Welfare, Government of India. Available from: http://www.hivpolicy.org/biogs/HPE0016b.htm.

3. The clinical use of blood - Handbook. Blood Transfusion safety 2002, World Health Organization, Geneva. Available from: https:// apps.who.int/dsa/cat98/blood8.htm.

4. Srikrishna A, Sitalakshmi S, Damodar P. How safe are our safe donors? Indian J. Pathol Microbiol 1999;42:411-6.

5. Siddiqui FA, Akhtar K, Sherwani RK, Rehman K, Alam F, Ansari A. Prevalence of Hepatitis C virus in Aligarh: A seven year experience. Indian J Comm Med 2009;34:264-5.

6. Makroo RN, Salil P, Vashist RP, Shivlal. Trend of HIV infection in the blood donors of Delhi. Indian J. Pathol Microbiol 1996;39:13942 .

7. Kurl A, Berry V, Dhanoa J, Masih A. Sero-positivity of HBsAg, Anti HCV and Anti HIV among blood donors: A comparative study on three years of 5 years interval. Indian J Pub Health 2007;51:41-2.

8. Adhikari L, Bhatta D, Tsering DC, Sharma DK, Pal R, Gupta A Infectious disease markers in blood donors at Central Referrel Hospital, Gangtok, Sikkim. Asian J Transf Sci 2010;4:41-2.

9. Sood S, Malvankar S. Seroprevalence of Hepatitis B Surface Antigen, Antibodies to the Hepatitis Virus, and Human Immunodeficiency Virus in a Hospital-Based Population in Jaipur, Rajasthan. Indian J Comm Med 2010;35:165-8.

10. Tiwari BR, Ghimire P, Kandel SR, Rajkarnikar M. Seroprevalence of HBV and HCV in blood donors: A study from regional blood transfusion services of Nepal. Asian J Transf Sci 2010;04:91-3.

11. Shukla RS, Bhuyan KK. Can data on HIV seropositivity among blood donors provide an insight into HIV prevalence in the General Population. Indian J Pub Health 2007;51:14-21.

12. Bagga PK, Singh SP. Seroprevalence of Hepatitis C antibodies in healthy blood donors - a propective study. Indian J Pathol Microbiol 2007;50:429-32.

13. Panda M, Kar K. HIV, Hepatitis B and C infection status of the blood donors in a blood bank of Tertiary Health Care Centre of Orissa. Indian J Pub Health 2008;52:43-4. 